\title{
Acute kidney injury in cancer patients and impedance cardiography-assisted renal replacement therapy: Experience from the onconephrology unit of a Chinese tertiary hospital
}

\author{
TAO WANG ${ }^{1}$, YAN ZHANG ${ }^{2}$, QINGXIA LI ${ }^{3}$, SHUMIN JIA $^{4}$, CHANGJIN SHI $^{5}$, KAI NIU $^{1}$ and BING LIU ${ }^{1}$ \\ ${ }^{1}$ Department of Nephrology, Hebei General Hospital, Shijiazhuang, Hebei 050051; ${ }^{2}$ Department of Dermatology, \\ Hebei Provincial Tumor Hospital, Shijiazhuang, Hebei 050011; ${ }^{3}$ Department of Oncology; ${ }^{4}$ Hemodialysis Center, \\ Hebei General Hospital, Shijiazhuang, Hebei 050051; ${ }^{5}$ Department of Urology, Hebei Provincial Tumor Hospital, \\ Shijiazhuang, Hebei 050011, P.R. China
}

Received April 7, 2017; Accepted July 27, 2017

DOI: $10.3892 /$ etm.2017.5244

\begin{abstract}
Acute kidney injury (AKI) in cancer patients may disrupt anticarcinogenic treatment and greatly increase associated mortality rates. The present study reported on the management of cancer patients with AKI and, from the nephrologic viewpoint, on the significance of fine volume control during the continuous renal replacement therapy (CRRT). The records of 117 cancer patients with AKI treated over three years were reviewed and their data were compared with those of 120 healthy controls. AKI was defined according to the Kidney Disease: Improving Global Outcomes criteria with serum creatinine levels determined on initial admission and for the diagnosis of AKI. CRRT with concomitant impedance cardiography (ICG) monitoring was performed in 79 patients. On average, AKI manifested as a $1.68 \pm 0.38$-fold increase in serum creatinine within $10.2 \pm 5.7$ days. The causes of AKI were diverse, with the major ones being nephrotoxic agents, hypotension and obstructive nephropathy. Renal biopsy confirmed two cases of thrombotic microangiopathy, due to the use of interfon- $\alpha$ and sunitinib malate, respectively, and a third case of cast nephropathy caused by immunoglobulin D multiple myeloma. The patients were generally marantic and had compromised cardiac function compared with the healthy controls. The CRRT prescription was discriminatingly optimized by the ICG parameters effecting discreet fluid balance, as the thoracic fluid content was significantly correlated with the ultrafiltration rate. By considering the causative mechanisms and applying subtle ICG-assisted volume control, the present study may thus help to
\end{abstract}

Correspondence to: Professor Bing Liu, Department of Nephrology, Hebei General Hospital, 348 West Heping Boulevard, Shijiazhuang, Hebei 050051, P.R. China

E-mail: nephrology2009@hotmail.com

Key words: acute kidney injury, cancer, continuous renal replacement therapy, hemodynamic monitoring, impedance cardiography improve the safety and efficacy of CRRT in cancer patients with AKI. In addition, it provided information to bring advances in onconephrology, a novel nephrological subspecialty field.

\section{Introduction}

Acute kidney injury (AKI) is a particularly common complication in cancer patients and associated with increased mortality (1). It may occur as a direct or indirect consequence of the cancer itself, its treatment or associated complications $(2,3)$. Among the heterogeneous etiology, nephrotoxic AKI by chemotherapeutic drugs is one of the most frequently encountered in malignancies (4), whereas in rare cases, renal infiltration in chronic myeloid leukemia (CML) has also been reported (5). Under these circumstances, renal hypoperfusion further jeopardizes the patients to the development of AKI or more likely exacerbates it to full acute renal failure (ARF) (3). On the other hand, patients with AKI are predisposed to fluid overload, which may also increase mortality (6). Maintenance of an appropriate fluid balance is hence considered vital to the management of AKI in cancer patients, in which AKI usually emerges in the context of multiple organ dysfunction (7).

More recently, it has been shown that the presence of an underlying cancer alone should no longer deny the critically ill patients the chance of receiving continuous renal replacement therapy (CRRT) or other advanced life-support measures $(8,9)$. Continuous venovenous hemodiafiltration (CVVHDF) with its dialysis component is thought to be the best option to treat ARF in cancer patients (10). CRRT, however, is not a panacea without concern. For instance, one obvious potential well-recognized hazard is overly aggressive fluid removal evoking hypotension, renal hypoperfusion, prerenal azotemia, and de novo renal injury or even ARF (11). An inaccurate volume assessment, if left unattended, may thus lead to the inappropriate implementation of therapy and the clinical consequence may be costly. Therefore, careful assessment of the fluid status prior to the initiation of and during CRRT is required. In this regard, impedance cardiography (ICG) is a subtle and non-invasive method of evaluating hemodynamic parameters (12) and ICG-guided CVVHDF was of bona fide 
value in a previous study by our group on the management of CML-associated ARF (5).

With increasingly complex cancer treatment protocols, novel antineoplastic drugs and a growing emphasis on AKI in cancer patients, onconephrology has evolved into a new field of nephrology (13). It is an intersection between nephrology and oncology, in which nephrologists are required to be familiar with the epidemiology, etiology and treatment of AKI in cancer patients. As such, this concept of onconephrology was put into practice and the present study reported on recent clinical experience in this novel sub-specialty field of nephrology. The study also focused on three rare cases of biopsy-confirmed AKI in patients with various types of malignancy.

\section{Materials and methods}

Subjects. The clinical records of 117 cancer patients with AKI that were either admitted or referred to the onconephrology unit between February 2014 and December 2016 at Hebei General Hospital, a tertiary medical center in Shijiazhuang (China), were reviewed. They were found to have irresectable tumors and $93(79.5 \%)$ received chemotherapy. The onset of AKI was defined by the Kidney Disease: Improving Global Outcomes criteria (14). At the first presentation, laboratory tests were performed according to standard procedures, followed by electrocardiogram, echocardiography, renal ultrasound and other auxiliary examinations when deemed necessary. Patients with overt congestive heart failure were excluded. The estimated glomerular filtration rate (eGFR) was deduced from the modified version of the Modification of Diet in Renal Disease (MDRD) equation for Chinese individuals: $\mathrm{eGFR}=175 \mathrm{x}$ Serum creatinine $^{-1.234} \mathrm{x}$ age $^{-0.197}$ (x0.79 for females). A total of 120 age-matched 'healthy' controls were recruited later during annual health examinations. The term 'healthy' was introduced for the descriptive purpose against the existence of cancer. They went through an extensive work-up, including hematological screening tests, urinalysis, chest X-ray and the abovementioned examinations. These subjects were non-diabetic without edema, free of cardiac and renal diseases, devoid of health problems requiring immediate medical attention and received voluntary referral ICG testing. Other selection criteria were similar to those in a previous study by our group (15). Data from these healthy controls were used for establishing the baseline impedance as well as the magnitude and duration of the change in pertinent parameters. The study protocol was approved by the institutional review board and written informed consent was obtained from all participants or their authorized kin.

Etiology of AKI. The cause of AKI was estimated collectively by three senior nephrologists. Depending upon the patient's condition and consent, ultrasound-guided percutaneous renal biopsy was performed under local anesthesia. The specimen was subjected to light, fluorescent and electron microscopy examinations. Immunofixation electrophoresis was used when multiple myeloma was suspected. During the fixation phase, antiserum was inoculated with the serum samples and the presence of monoclonal protein could be detected in the form of precipitant (16).
$C R R T$ and ICG. In addition to the symptom-oriented treatments, 79 patients accepted CRRT. It was performed with an Aquarius machine (Aquarius; Edwards Lifesciences, Maurepas, France) using polysulfone hemofilters (AV 600; Fresenius, Oberursel, Germany) through a provisional venous catheter. Substitution solutions were delivered pre-filter at a rate of $35 \mathrm{ml} / \mathrm{kg} / \mathrm{h}$. The ICG (BioZ, CardioDynamics, CA, USA) was performed as previously described $(5,12)$ during CRRT (initiation, for $5 \mathrm{~h}$ thereafter and cessation) and in the healthy controls. The ultrafiltration was performed in a linear decreasing manner (17) and adjusted according to the ICG data, particularly the thoracic fluid content (TFC). To evaluate the utility of ICG in hemodynamic monitoring during the CRRT, the healthy controls and cancer patients were then stratified according to two ICG parameters, the stroke index (SI) and TFC. This generated a plot of four quadrants with the respective cut-off point for the SI and TFC as $35 \mathrm{ml} / \mathrm{m}^{2}$ and $35 \mathrm{kOhm}^{-1}$.

Statistical analysis. Statistical analyses were performed with the SPSS package (version 19.0; IBM Corp., Armonk, NY, USA). Values are expressed as the mean \pm standard deviation. Significantly skewed data were log-transformed prior to the analysis. Analysis of variance with Bonferroni's post-hoc test, Student's t-test and the $\chi^{2}$ test were performed to assess differences between groups for continuous and categorical variables, respectively. Correlations were examined using Pearson's test. $\mathrm{P}<0.05$ was considered to indicate a statistically significant difference.

\section{Results}

Epidemiology of AKI in cancer patients. The clinical profiles of the participants were listed in Table I. Comparing with the healthy controls, the cancer patients were in worse condition with a lower body mass index, hypotension, overt anemia, elevated serum creatinine ( $\mathrm{Scr}$ ), decreased eGFR, hypoalbuminemia and hypolipidemia. However, the Scr and eGFR were $106.1 \pm 37.8 \mu \mathrm{mol} / 1$ and $76.4 \pm 22.1 \mathrm{ml} / \mathrm{min} / 1.73 \mathrm{~m}^{2}$, respectively, in the cancer patients prior to the onset of AKI. On average, the increase of Scr was 1.68 \pm 0.38 -fold within 10.2 \pm 5.7 days. Sites of primary malignancy are presented in Table II with the digestive, respiratory and hematological systems as the top three sites. As illustrated in Table III, the causes of AKI were diverse and the leading ones were nephrotoxic agents, hypotension (grouped with extracellular dehydration) and obstructive nephropathy.

Hemodynamic features prior to CRRT. Compared with the healthy controls (Table IV), the pre-CRRT data of the patients presented a significantly higher heart rate, system vascular resistance index (SVRI), TFC and systolic time ratio (STR). Furthermore, markedly lower blood pressures, cardiac index (CI), SI and velocity index (VI) were recorded. The markedly decreased SI, VI and prolonged STR may indicate compromised cardiac function of myocardial origin.

Evaluation of CRRT. CRRT was performed with individualized ultrafiltration prescription in the light of the ICG data. Changes in the TFC were inversely correlated with the ultrafiltration rate $(\mathrm{r}=0.87, \mathrm{P}<0.01$; Fig. 1$)$. In patients with 
Table I. Clinical profiles between the healthy controls and cancer patients with acute kidney injury.

\begin{tabular}{lcc}
\hline Parameter & Healthy controls & Cancer patients \\
\hline Subjects & 120 & 117 \\
Age (years) & $60.6 \pm 11.9$ & $61.7 \pm 10.5$ \\
Gender (male/female) & $80 / 40$ & $76 / 41$ \\
Body mass index $\left(\mathrm{kg} / \mathrm{m}^{2}\right)$ & $22.7 \pm 3.2$ & $18.9 \pm 2.7^{\mathrm{a}}$ \\
Systolic blood pressure $(\mathrm{mmHg})$ & $132.7 \pm 13.3$ & $112.1 \pm 20.8^{\mathrm{a}}$ \\
Diastolic blood pressure $(\mathrm{mmHg})$ & $79.0 \pm 12.5$ & $63.1 \pm 15.2^{\mathrm{a}}$ \\
White blood cell count $\left(\mathrm{x} 10^{9} / \mathrm{l}\right)$ & $7.3 \pm 2.1$ & $8.5 \pm 3.7$ \\
Neutrophils $(\%)$ & $69.7 \pm 10.6$ & $76.7 \pm 23.1$ \\
Hemoglobin concentration $(\mathrm{g} / \mathrm{l})$ & $136.7 \pm 16.2$ & $89.3 \pm 14.5^{\mathrm{a}}$ \\
Glutamic pyruvic transaminase $(\mathrm{IU})^{\mathrm{b}}$ & $22.6 \pm 10.0$ & $20.1 \pm 14.0$ \\
Serum creatinine $(\mu \mathrm{mol} / \mathrm{l})^{\mathrm{b}}$ & $79.8 \pm 22.3$ & $179.3 \pm 38.4^{\mathrm{a}}$ \\
eGFR $\left(\mathrm{ml} / \mathrm{min} / 1.73 \mathrm{~m}^{2}\right)$ & $109.3 \pm 10.5$ & $35.0 \pm 21.2^{\mathrm{a}}$ \\
Serum potassium $(\mathrm{mmol} / \mathrm{l})$ & $4.1 \pm 1.2$ & $5.0 \pm 0.9$ \\
Fasting plasma glucose $(\mathrm{mg} / \mathrm{dl})^{\mathrm{b}}$ & $95.9 \pm 15.0$ & $87.2 \pm 16.4$ \\
Albumin $(\mathrm{g} / \mathrm{l})$ & $45.3 \pm 4.5$ & $25.9 \pm 5.3^{\mathrm{a}}$ \\
Total cholesterol $(\mathrm{mmol} / \mathrm{l})$ & $6.74 \pm 1.43$ & $3.49 \pm 1.00^{\mathrm{a}}$ \\
Triglycerides $(\mathrm{mmol} / \mathrm{l})^{\mathrm{b}}$ & $2.78 \pm 1.20$ & $1.37 \pm 0.81^{\mathrm{a}}$ \\
Fibrinogen $(\mathrm{g} / \mathrm{l})^{\mathrm{b}}$ & $3.39 \pm 0.78$ & $4.21 \pm 0.98$ \\
\hline
\end{tabular}

Values are expressed as the mean \pm standard deviation or as $n$. Differences between the two populations were examined by the Student's $t$-test or $\chi^{2}$ test when deemed appropriate. ${ }^{a} \mathrm{P}<0.05$; ${ }^{b} \log$-transformed values used in the analysis. eGFR, estimated glomerular filtration rate.

Table II. Types of primary malignancy within the cohort.

\begin{tabular}{lc}
\hline Site/type of malignancy & $\mathrm{n}(\%)$ \\
\hline Digestive system & $51(43.5)$ \\
Respiratory system & $29(24.8)$ \\
Hematology & $15(12.9)$ \\
Gynaecology & $10(8.5)$ \\
Urology & $9(7.7)$ \\
Miscellaneous & $3(2.6)$ \\
Total & $117(100)$
\end{tabular}

Miscellaneous types include one case of gastric mesothelioma, hemangiopericytoma and unknown primany tumor.

increased TFC, fluid was removed accordingly, while the other parameters were concurrently monitored. SI was significantly increased and SVRI decreased at $5 \mathrm{~h}$ after the initiation of CRRT. At the end of this procedure, the patients presented with major improvements, including increased systolic and diastolic blood pressure, CI, SI and VI, as well as reduced TFC and SVRI. Similarly, the central venous pressure was significantly decreased from 17.0 \pm 2.9 to $11.0 \pm 4.1 \mathrm{mmH}_{2} \mathrm{O}$. The efficacy of the CRRT was then validated in Fig. 2 . An $\mathrm{SI}>35 \mathrm{ml} / \mathrm{m}^{2}$ and $\mathrm{TFC} \leq 35 \mathrm{kOhm}^{-1}$ was denoted as a hemodynamically stable group (Fig. 2A) and vice versa for the hemodynamically unstable one (Fig. 2D). The remaining two groups were deemed to have an intermediate risk (Fig. 2B and C). In total, $70.0 \%$ of the healthy
Table III. Causes of acute kidney injury in the cancer patients.

\begin{tabular}{lc}
\hline Cause & $\mathrm{n}(\%)$ \\
\hline Prerenal & \\
Capillary leak syndrome (interleukin-2) & $1(0.86)$ \\
Drugs (ACEI, NSAIDs) & $6(5.13)$ \\
Extracellular dehydration (diarrhea and vomiting) & $10(8.55)$ \\
Hepatorenal syndrome & $1(0.86)$ \\
Hypotension & $21(17.95)$ \\
Sepsis & $6(5.13)$ \\
Renal & \\
Hypercalcemia & $2(1.71)$ \\
Intravascular hemolysis & $1(0.86)$ \\
Myeloma kidney & $5(4.27)$ \\
Nephrotoxic agents & $45(38.46)$ \\
Thrombotic microangiopathy & $2(1.71)$ \\
Tumor infiltration & $3(2.56)$ \\
Tumor lysis syndrome & $3(2.56)$ \\
Postrenal & \\
Ureteral or bladder outlet occlusion & $11(9.4)$ \\
Total & $117(100)$ \\
\hline
\end{tabular}

ACEI, angiotensin converting enzyme inhibitors; NSAIDs, non-steroid anti-inflammatory drugs.

controls, $0 \%$ of the cancer patients prior to CRRT and $34.2 \%$ of them after the CRRT were in the stable group, whereas 
Table IV. Hemodynamic parameters by impedance cardiography in healthy controls and cancer patients.

\begin{tabular}{|c|c|c|c|c|c|}
\hline Parameter & Healthy controls & Pre-CRRT & $5 \mathrm{~h}$ of CRRT & Post-CRRT & Normal range \\
\hline $\mathrm{n}$ & 80 & 79 & 79 & 79 & - \\
\hline HR (beats/min) & $76.1 \pm 15.3$ & $89.2 \pm 13.4^{\mathrm{a}}$ & $85.8 \pm 11.5$ & $83.1 \pm 10.1$ & $58-86$ \\
\hline $\mathrm{SBP}(\mathrm{mmHg})$ & $136.0 \pm 21.8$ & $110.6 \pm 23.1^{\mathrm{a}}$ & $114.6 \pm 18.1$ & $123.6 \pm 17.7^{b}$ & $100-140$ \\
\hline $\mathrm{DBP}(\mathrm{mmHg})$ & $77.6 \pm 12.2$ & $61.5 \pm 17.3^{\mathrm{a}}$ & $64.3 \pm 12.5$ & $73.4 \pm 10.5^{\mathrm{b}}$ & $60-90$ \\
\hline $\mathrm{CI}\left(\mathrm{l} / \mathrm{min} / \mathrm{m}^{2}\right)$ & $3.02 \pm 0.57$ & $2.30 \pm 0.61^{\mathrm{a}}$ & $2.58 \pm 0.46$ & $2.87 \pm 0.55^{\mathrm{b}}$ & $2.5-4.2$ \\
\hline $\mathrm{SI}\left(\mathrm{ml} / \mathrm{m}^{2}\right)$ & $47.16 \pm 8.93$ & $25.1 \pm 7.95^{\mathrm{a}}$ & $30.0 \pm 7.2^{\mathrm{b}}$ & $36.1 \pm 7.50^{\mathrm{b}}$ & $35-65$ \\
\hline SVRI (dyne.sec $/ \mathrm{cm}^{5} / \mathrm{m}^{2}$ ) & $1,573.5 \pm 332.3$ & $2,934.8 \pm 908.8^{a}$ & $2,252.5 \pm 733.0^{\mathrm{b}}$ & $1,901.3 \pm 401.6^{\mathrm{b}}$ & $1,337-2,483$ \\
\hline $\mathrm{TFC}(/ \mathrm{k} \Omega)$ & $35.30 \pm 7.71$ & $53.5 \pm 11.9^{\mathrm{a}}$ & $49.5 \pm 19.8$ & $41.7 \pm 11.5^{\mathrm{b}}$ & $30.0-50.0$ \\
\hline VI $(/ 1,000 \mathrm{sec})$ & $47.62 \pm 12.76$ & $28.7 \pm 5.3^{\mathrm{a}}$ & $30.2 \pm 10.1$ & $35.8 \pm 7.8^{b}$ & $33-65$ \\
\hline STR & $0.33 \pm 0.08$ & $0.49 \pm 0.11^{\mathrm{a}}$ & $0.45 \pm 0.13$ & $0.38 \pm 0.17$ & $0.30-0.50$ \\
\hline
\end{tabular}

${ }^{\mathrm{a}} \mathrm{P}<0.05$ significantly different by unpaired t-test between the healthy controls and cancer patients prior to CRRT. ${ }^{\text {b }}<0.05$, significantly different by analysis of variance against the pre-CRRT values. CRRT, continuous renal replacement therapy; HR, heart rate; SBP, systolic blood pressure; DBP, diastolic blood pressure; CI, cardiac index; SI, stroke index; SVRI, system vascular resistance index; TFC, thoracic fluid content; VI, velocity index; STR, systolic time ratio.

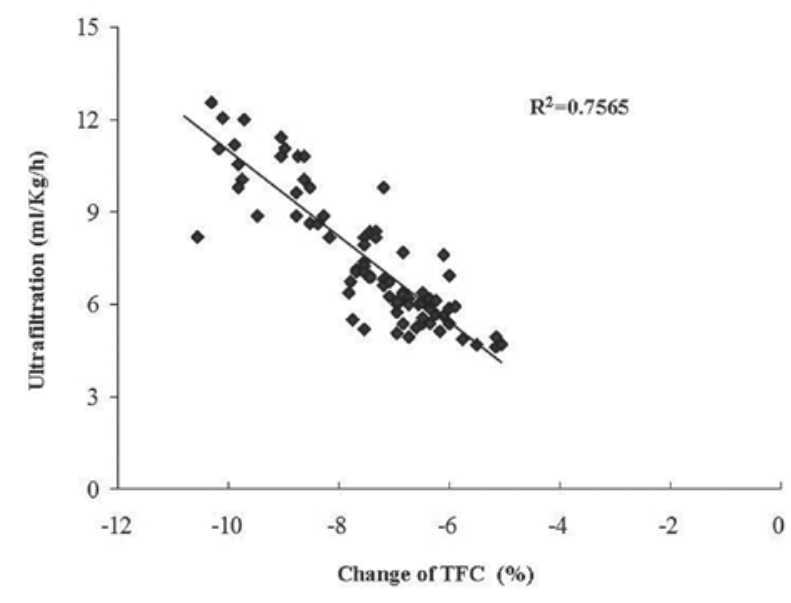

Figure 1. Association between ultrafiltation rate and percentage change of TFC during continuous renal replacement therapy. TFC, thoracic fluid content.

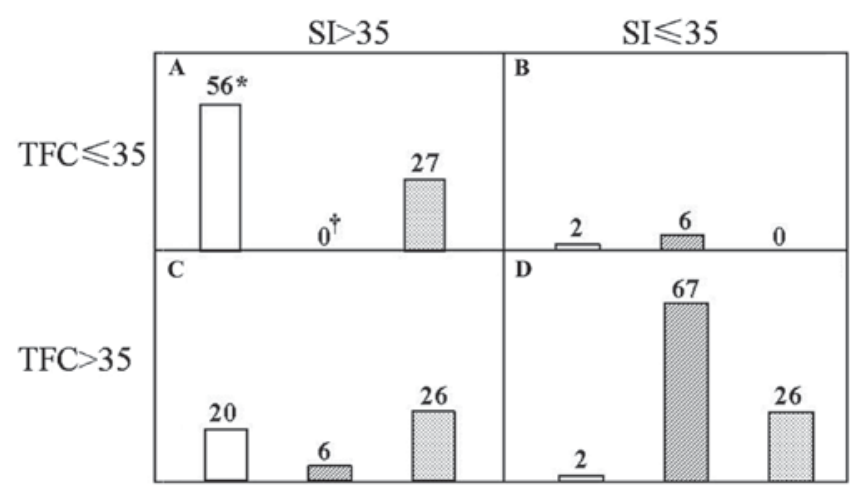

Figure 2. Utility of the impedance cardiography during the CRRT. Subjects were categorized into one of four hemodynamic quadrants according to SI and TFC. (A) SI $>35$ and TFC $\leq 35$; (B) SI $\leq 35$ and TFC $\leq 35$; (C) SI $>35$ and TFC $>35$; (D) $\mathrm{SI} \leq 35$ and TFC $>35$. For each quadrant, the open, hatched and dotted columns represent the healthy controls, and cancer patients at the initiation and at the end of the CRRT, respectively. The number of the subjects was denoted on top of the individual columns. ${ }^{*} \mathrm{P}<0.05$ vs. pre- or post-CRRT value; ${ }^{\dagger} \mathrm{P}<0.05$ vs. post-CRRT values. CRRT, continuous renal replacement therapy; TFC, thoracic fluid content; SI, stroke index. the corresponding rates were $2.5,84.8$ and $32.9 \%$ in the unstable group, respectively. Of note, the differences between any two groups were significant among the control, pre-and post-CRRT values.

Therapy-induced thrombotic microangiopathy (TMA). TMA occurred in a patient with chronic myeloid leukemia at the end of 1 year of interfon- $\alpha$ therapy (Fig. 3A and B) and in a patient with gastric mesothelioma at the end of 6 months of sunitinib malate treatment (Fig. 3C and D). In each of the two cases, diffuse and severe endothelial damage with double contours and occlusion of capillary lumen was observed. Nodular expansion of mesangium was also seen (Fig. 3A and C). Each of the two patients was negative on immunofluorescence analysis (data not shown) and electron microscopy of the glomerular capillary wall revealed expansion of the subendothelial space by electron-lucent debris (Fig. 3B and D).

Cast nephropathy in immunoglobulin D myeloma. Analysis of bone marrow aspirate revealed $12.5 \%$ of plasma cells in a patient referred to us whom we later diagnosed with multiple myeloma. Based on the first biopsy he received elsewhere 3 months earlier, the patient was initially considered to have an ischemic lesion (Fig. 4A). The second biopsy was performed at our hospital and cast nephropathy was identified (Fig. 4B and C). Immunofixation electrophoresis then clearly detected immunoglobulin (Ig) D and light chain $\lambda$ precipitation (Fig. 4D).

\section{Discussion}

The present study identified that cancer patients may develop AKI when presenting with a decrease in Scr within a short period and a deterioration of the general condition is thought to enhance the susceptibility (4). The altered ICG parameters further revealed that AKI was accompanied by circulatory detriments, which improved substantially after CRRT. In addition, a change of TFC during CRRT was significantly correlated with the ultrafiltration rate. Furthermore, AKI was 

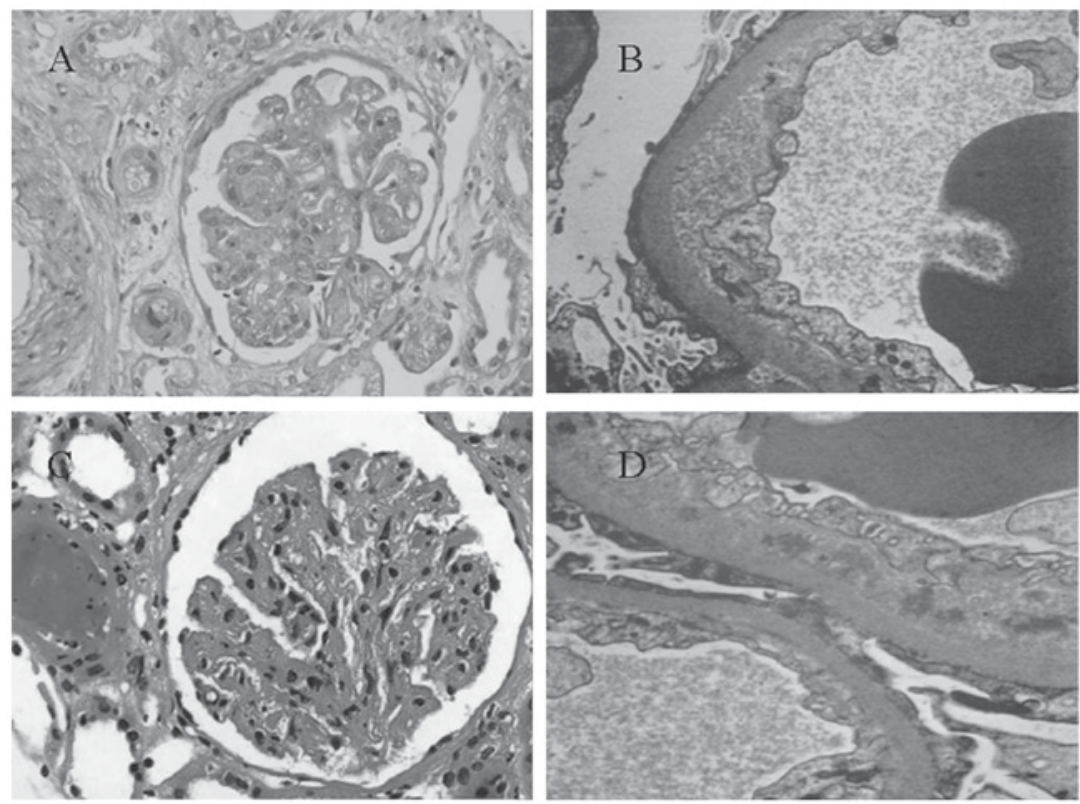

Figure 3. Thrombotic microangiopathy in patients receiving (A and B) interferon- $\alpha$ or (C and D) sunitinib malate. (A and C) Representative light microscopy images demonstrating that the glomerulus presented with global thickening of the glomerular basement membranes by glassy eosinophilic material associated with segmental mesangial interposition. Nodular expansion of mesangium and occlusion of capillary lumens without mesangial hypercellularity was observed (magnification, x200). (B and D) Electron microscopy revealed swollen endothelium with wavy inner contours, expansion of the subendothelial space by electron lucent debris and loss of endothelial fenestrate (magnification, x2,000).
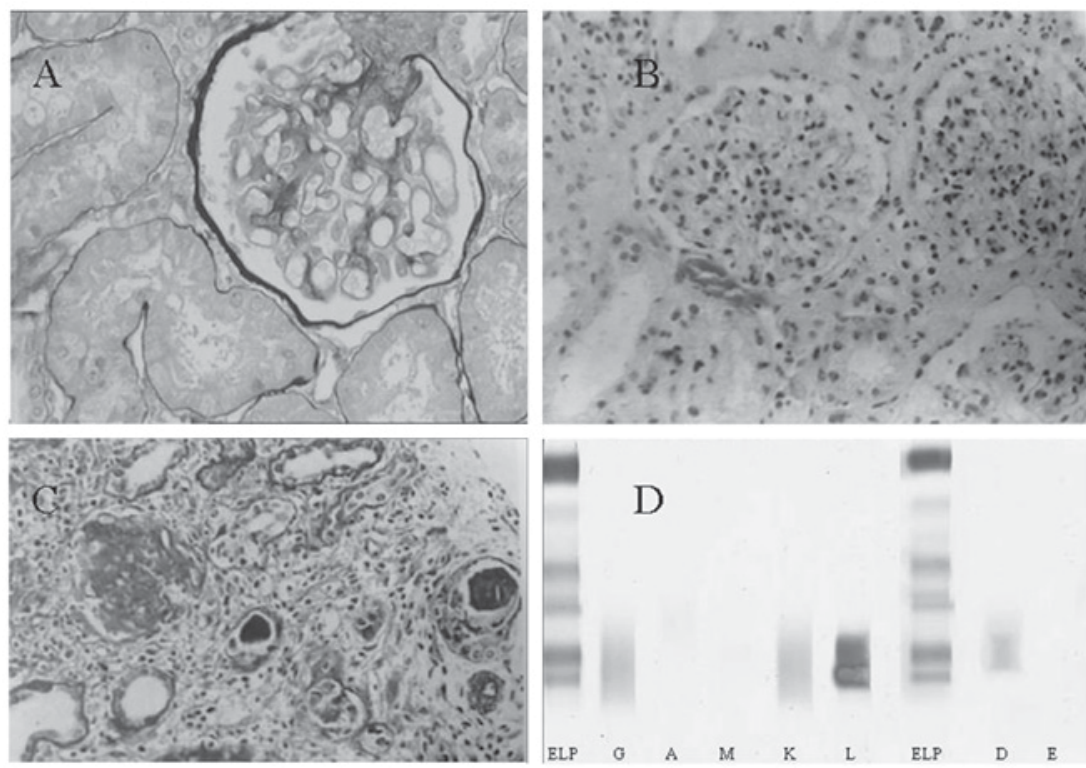

Figure 4. Cast nephropathy and serum immunofixation electrophoresis in IgD multiple myeloma. (A) Slightly corrugated capillary loop and turgescence of the capsule (magnification, x200). (B) Corrugated capillary loop and increased mesangium with glomerular hypercellularity (magnification, x100). (C) Formation of protein casts with interstitial infiltration of inflammatory cells (magnification, x100). (D) Immunofixation electrophoresis of serum revealed a restricted IgD and $\lambda$ band. ELP, reference lane representing albumin, $\alpha-, \beta$ - and $\gamma$-globulin from the top down. IgD, immunoglobulin D.

more likely in patients with a malignancy of the digestive, respiratory and hematological system, in general agreement with a previous study reporting that patients with liver cancer and multiple myeloma were more susceptible to AKI (18).

The onset of AKI in cancer patients greatly increases the short-term risk of mortality (13). Furthermore, delayed nephrology consultation of patients with ARF is also associated with increased mortality (19). At the same time, there is a paucity of information regarding the non-invasive hemodynamic monitoring of cancer patients with renal complications, particularly those requiring CRRT. The onconephrology unit, with higher competence in oncology and nephrology, may be called upon to services as recently advocated by Thajudeen and Salahudeen (20). In this respect, the present study may be of interest to clinicians in general and nephrologists in particular.

Cancer patients with AKI are often hemodynamically unstable (10). This was proved by the different ICG data 
between the healthy controls and the cancer patients. Dynamic and vigilant monitoring of blood volume is thus conceivably imperative. The ICG could readily meet this requirement by yielding a broad panel of parameters that reflect blood flow, systemic vascular resistance, myocardial contractility and fluid status. While the empirical assessment of ultrafiltration using the dry weight is error-prone with a deviation of 50\% (21) and detection of non-specific symptoms via small undulations of volume is far less sensitive, the good correlation of the changes in TFC with the ultrafiltration rate allows for 'fine-tuning' the latter during CRRT. Combined with the SI, it may also be used for evaluating the outcomes of CRRT. After CRRT, the stable, intermediate and unstable groups each accounted for one third of the cancer patients. Their risk features indicated that these groups may respectively require close monitoring, further volume removal and extra CRRT sessions with possible isotropic agents. These combined analyses were hence prognostically more powerful and discriminating than any clinical assessment considered alone. Careful interpretation of the data during CRRT may allow the clinician to detect the emergence of potentially adverse hemodynamic fluctuations, particularly during periods of apparent clinical stability, and optimize the ultrafiltration prescription.

A better understanding of the etiology of AKI in cancer patients may help expedite the diagnosis and treatment. The mechanisms of AKI in cancer are diverse in origin: Infiltration (22) and urinary tract obstruction (23) due to the cancer per se; acute tumor lysis syndrome (24) and nephrotoxicity (25) caused by the treatment; sepsis (2) and hypercalcemia (26) associated with severe complications. With rapid advances in cancer therapy, chemotherapy-induced AKI has drawn increasing attention (4). Indeed, AKI may occur in up to $30 \%$ of cancer patients receiving cisplatin (27). The diversity in the etiology was exemplified in the cases of the present study. Of note, chemotherapy was not only the leading cause of AKI in the cohort of the present study, but was observed in nearly one half of the patients who received anticancer treatment (45 out of 93). This high prevalence may be due to the fact that a lesser amount of patients without AKI were followed up. As modern oncology has been rapidly turning into a multidisciplinary service, clinicians should be familiar with these cancer-associated aspects prior to making appropriate prophylactic and therapeutic decisions.

The present study observed three biopsy-confirmed rare cases: TMA in a patient with CML taking interfon- $\alpha$ and in a patient with gastric mesothelioma taking sunitinib malate. The mechanisms by which interfon- $\alpha$ induced TMA remain elusive but are thought to be an immune-mediated process (28). It is noteworthy that the underlying CML may also be contributory, since chronic hepatitis $\mathrm{C}$ patients receiving interfon- $\alpha$ seldom develop TMA. Sunitinib malate is a novel tyrosine kinase inhibitor that simultaneously abrogates the function of nitric oxide synthetase and vascular endothelial growth factor (VEGF). The action of VEGF-targeted therapy may impair the integrity of renal endothelium and lead to the resultant TMA. In both cases, double contours and nodular expansion of mesangium may be seen, indicating rather chronic changes. Therefore, close monitoring of renal function should be mandatory, particularly in patients on long-term inferton- $\alpha$ use or VEGF inhibition.
AKI caused by lymphomatous infiltration of the kidneys, cast nephropathy in multiple myeloma or tumor lysis syndrome are unique to the cancer population. IgD multiple myeloma represents $\sim 2 \%$ of multiple myeloma cases with an incidence of renal involvement of $70 \%$ (29). By contrast, these numbers in IgG multiple myeloma are 59 and $15 \%$, respectively. Patients are usually younger than those with $\operatorname{IgG}$ or $\operatorname{IgA}$ myeloma and are more likely to present with nonspecific systemic symptoms, a more rapid progressive clinical course, a higher rate of extramedullary involvement and shorter survival time $(30,31)$. Unfortunately, the majority of clinical laboratories do not include $\operatorname{IgD}$ in the initial workup of monoclonal gammopathies (32). It is prudent for clinicians to test for $\operatorname{IgD}$ and $\operatorname{IgE}$ when cast nephropathy is suspected or serum light chain levels demonstrate a pronounced elevation without a corresponding up-shift of monoclonal $\operatorname{IgG}, \operatorname{IgA}$ and $\operatorname{IgM}$, as in the case of the present study.

In conclusion, the present study highlighted the fact that AKI in cancer patients may have different underlying mechanisms, manifest as a discrepant volume status and require distinct fluid therapy. As such, the introduction of ICG may improve the safety and efficacy of CRRT in these patients, particularly those with a cardiac comorbidity. The establishment of onconephrology may assuredly answer the challenges of expeditious diagnosis and effective management of cancer patients with AKI.

\section{Acknowledgements}

The authors thank Iain Forem (Bournemouth, UK) for English language editing. This study was supported by a grant (no. 20170315) from the Health and Family Planning Commission of Hebei Province.

\section{References}

1. Lameier NH, Flombaum CD, Moreau D and Ronco C: Acute renal failure in cancer patients. Ann Med 37: 13-25, 2005.

2. Finkel KW and Foringer JR: Renal disease in patients with cancer. Nat Clin Pract Nephrol 3: 669-678, 2007.

3. Humphreys BD, Soiffer RJ and Magee CC: Renal failure associated with cancer and its treatment: An update. J Am Soc Nephrol 16: 151-161, 2005.

4. Perazella MA and Moeckel GW: Nephrotoxicity from chemotherapeutic agents: Clinical manifestations, pathobiology, and prevention/therapy. Semin Nephrol 30: 570-581, 2010.

5. Wang T, Ma HC, Bai YL, Zhang JX and Xu JS: Continuous venovenous hemodiafiltration, impedance cardiography and critical care nephrology: A case study of chronic myeloid leukemia-associated acute renal failure. Crit Care Shock 14: 19-23, 2011.

6. Yerram P, Karuparthi PR and Misra M: Fluid overload and acute kidney injury. Hemodial Int 14: 348-354, 2010.

7. Soares M, Salluh JI, Carvalho MS, Darmon M, Ronco JR and Spector N: Prognosis of critically ill patients with cancer and acute renal dysfunction. J Clin Oncol 24: 4003-4010, 2006.

8. Benoit DD, Hoste EA, Depuydt PO, Offner FC, Lameire NH, Vandewoude KH, Dhondt AW, Noens LA and Decruyenaere JM: Outcome in critically ill medical patients treated with renal replacement therapy for acute renal failure: Comparison between patients with and those without haematological malignancies. Nephrol Dial Transplant 20: 552-558, 2005.

9. Darmon M, Thiery G, Ciroldi M, Porcher R, Schlemmer B and Azoulay E: Should dialysis be offered to cancer patients with acute kidney injury? Intensive Care Med 33: 765-772, 2007.

10. Berghmans T, Meert AP, Markiewicz E and Sculier JP: Continuous venovenous haemofiltration in cancer patients with renal failure: A single-centre experience. Support Care Cancer 12: 306-311, 2004. 
11. Kazory A and Ross EA: Contemporary trends in the pharmacological and extracorporeal management of heart failure: A nephrologic perspective. Circulation 117: 975-983, 2008.

12. Packer M, Abraham WT, Mehra MR, Yancy CW, Lawless CE, Mitchell JE, Smart FW, Bijou R, O'Connor CM, Massie BM, et al: Utility of impedance cardiography for the identification of short-term risk of clinical decompensation in stable patients with chronic heart failure. J Am Coll Cardiol 47: 2245-2252, 2006.

13. Salahudeen AK and Bonventre JV: Onconephrology: The latest frontier in the war against kidney disease. J Am Soc Nephrol 24: 26-30, 2013.

14. Kidney Disease: Improving Global Outcomes (KDIGO) Acute Kidney Injury Work Group: KDIGO clinical practice guideline for acute kidney injury. Kidney Int Suppl 2: 19-36, 2012.

15. Wang T, Zhang Y, Ma J, Feng Z, Niu K and Liu B: Additive effect of polymorphisms in the $\beta 2$-adrenoceptor and NADPH oxidase p22 phox genes contributes to the loss of estimated glomerular filtration rate in Chinese. Clin Exp Pharmacol Physiol 41: 657-662, 2014.

16. Dimopoulos M, Kyle R, Fermand JP, Rajkumar SV, San Miguel J, Chanan-Khan A, Ludwig H, Joshua D, Mehta J, Gertz M, et al: Consensus recommendations for standard investigative workup: Report of the international myeloma workshop consensus panel 3. Blood 117: 4701-4705, 2011.

17. Donauer J, Kölblin D, Bek M, Krause A and Böhler J: Ultrafiltration profiling and measurement of relative blood volume as strategies to reduce hemodialysis-related side effects. Am J Kidney Dis 36: 115-123, 2000.

18. Christiansen CF, Johansen MB, Langeberg WJ, Fryzek JP and Sørensen HT: Incidence of acute kidney injury in cancer patients: A Danish population-based cohort study. Eur J Intern Med 22: 399-406, 2011.

19. Mehta RL, McDonald B, Gabbai F, Pahl M,Farkas A, Pascual MT, Zhuang S, Kaplan RM and Chertow GM: Nephrology consultation in acute renal failure: Does timing matter? Am J Med 113: 456-461, 2002.
20. Thajudeen B and Salahudeen AK: Role of tolvaptan in the management of hyponatremia in patients with lung and other cancers: Current data and future perspectives. Cancer Manag Res 8: 105-114, 2016.

21. Jaeger JQ and Mehta RL: Assessment of dry weight in hemodialysis: An overview. J Am Soc Nephrol 10: 392-403, 1999.

22. Seo-Mayer P, Kenney B, McNamara J, Stein J and Moeckel GW: Hematuria and decreased kidney function as initial signs of acute B-cell lymphoblastic leukemia. Am J Kidney Dis 56: 1001-1005, 2010.

23. Segal A: A case of acute kidney injury due to complex, partial, multifocal ureteral strictures. Nat Clin Pract Nephrol 4: 102-108, 2008.

24. Pabla N and Dong Z: Cisplatin nephrotoxicity: Mechanisms and renoprotective strategies. Kidney Int 73: 994-1007, 2008.

25. Perazella MA: Renal vulnerability to drug toxicity. Clin J Am Soc Nephrol 4: 1275-1283, 2009.

26. Miller RP, Tadagavadi RK, Ramesh G and Reeves WB: Mechanisms of cisplatin nephrotoxicity. Toxins (Basel) 2: 2490-2518, 2010.

27. de Jonge MJ and Verweij J: Renal toxicities of chemotherapy. Semin Oncol 33: 68-73, 2006.

28. Badid C, McGregor B, Faivre JM, Guerard A, Juillard L, Fouque D and Laville M. Renal thrombotic microangiography induced by interfon-alpha. Nephrol Dial Transplant 16: 846-848, 2001.

29. Kyle RA, Remstein ED, Therneau TM, Dispenzieri A, Kurtin PJ Hodnefield JM, Larson DR, Plevak MF, Jelinek DF, Fonseca R, et al: Clinical course and prognosis of smoldering (asymptomatic) multiple myeloma. N Engl J Med 356: 2582-2590, 2007.

30. Jancelewicz Z, Takatsuki K, Sugai S and Pruzanski W: IgD multiple myeloma: review of 133 cases. Arch Intern Med 135: 87-93, 1975.

31. Shimamoto Y, Anami Y and Yamaguchi M: A new risk grouping for IgD myeloma based on analysis of 165 Japanese patients. Eur J Haematol 47: 262-267, 1991.

32. Sinclair D and Cranfield T: IgD myeloma: A potential missed diagnosis. Ann Clin Biochem 38: 564-565, 2001. 